\title{
A Posture Recognition System to Track Drivers' Activities While Driving
}

\author{
Khaled Shaaban $^{a b} *$, Osama Halabi ${ }^{b}$, Eman Almughani $^{b}$ \\ ${ }^{a}$ Utah Valley University, Orem, United States, 84058 \\ ${ }^{b}$ Qatar University, Doha, Qatar, 2713
}

\begin{abstract}
To improve traffic safety, different systems were invented to warn drivers when detecting a conflict. However, drivers may be already aware of such conflicts. In these cases, warning drivers may not effective and may lead drivers to not pay attention to warnings in the case of serious conflicts. The purpose of this study is to develop a novel posture recognition system to detect different driver activities while driving. If drivers are not braking or decelerating in the case of a conflict, they are alerted and notified about the conflict. The proposed system is based on a passive method for measuring bodypostures by using two force sensor arrays to inspect the pressure patterns exhibited in the driver's seat and backrest. Different sensors' distributions were tested to find the most suitable distribution to improve the accuracy of the recognition. A virtual reality (VR) driving simulation was developed to test the accuracy of recognition in an immersive environment. Experiments were carried out to test the posture recognition accuracy in both realistic and VR settings. The results showed that the system could recognize different postures with high accuracy. Such a system can be used to alert drivers and disengage autonomous mode in the case of self-driving and autonomous vehicles when the situation unsafe.
\end{abstract}

Keywords: Sitting posture; activity recognition; driving simulator; virtual reality; autonomous vehicles

\section{Introduction}

There is always a need to develop advanced warning systems that can alert drivers in case of traffic conflicts. When designing such systems, one of the important factors is the frequency of warnings. If the warnings are false or too frequent, drivers can be disturbed, distracted, and may eventually ignore these

\footnotetext{
* Corresponding author. Tel.: (801) 863-8373

E-mail: kshaaban@uvu.edu

(C) 2021 International Association for Sharing Knowledge and Sustainability.

DOI: $10.5383 /$ JTTM.03.02.004
}

warnings. Therefore, there is a need for a system that classifies the need for warnings in different situations. The purpose of this study is to propose a new automated warning system. In this proposed system, once a potential conflict is detected, the system tracks the driver's reaction. If the driver is taking the proper reaction, no alert is needed. If the driver is not making any corrective action (decelerating, braking, etc.), a vibration is emitted depending on the risk assessment of the condition. The 
vibration intensity will vary according to the risk of the situation.

Typical distracting activities that drivers engage in while driving include dialing a cell phone, responding to a ringing cell phone, text messaging, adjusting radio/CD, music listening, and using navigation systems $[1,2]$. Being distracted with cell phones has been considered one of the main common types of distraction in recent times [3-5]. Multiple types of distractions can occur at the same time. Therefore, there is always a need to develop warning systems for drivers to react in the case of conflicts especially when distracted.

Different studies have focused on activity recognition using postures information. These studies covered several applications including medical, education, and traffic safety applications. Mota et. al. [6] carried out studies about postures recognition related to a children's interest level while performing a learning task. As part of the study, nine postures were recognized, such as leaning forward left, sitting upright, leaning back, etc. Zhu et al. [7] presented Euclidean distance metrics for measurement and matching of sitting postures as well as experiments and comparisons of different classification algorithms. Some studies found that the postures of drivers could provide informative information regarding drivers' activities [8,9]. To accomplish this task, force sensor arrays integrated inside the driver's seat and backrest fabric can be used to sense pressure on the seat and then analyze this data to predict the activity of the driver $[6,8$, 9]. Riener et al. [10] used the driver's body postures to reduce the risk of different driving situations by evaluating pressure patterns collected from force sensors arrays that integrated into the fabric of the driver seat and backrest.

Some studies have combined both sitting postures with a vibrotactile warning system to achieve a better intelligent driver assistance system. According to Higuchi and Raksincharoensak [11], adding a vibrotactile seat warning system for reducing the forward vehicle collision has a positive impact in reducing the forward collision risks. Van Erp et. al. [12] provided a tactile seat display and a visual display in a navigation system. Drivers' workloads and reaction time were measured. A decrease in the performance was realized when using the visual display, but the performance, while using tactile and multimodal displays, resulted in a faster reaction to navigation messages and reduced the mental effort of the drivers. Riener and Ferscha [13] designed a reconfigurable vibrotactile car seat for haptic interaction. Two types of mats, one for pressure sensing and another one for haptic stimulation. The size and place of vibrotactile notifications were reconfigured based on drivers' sitting postures. The pressure sensors were evaluated to ensure that the driver is capable to feel the full range of haptic feedback information.

Zheng et al. [14] explored the vibrotactile haptic feedback hypothesis but in a different application. He focused on sitting posture guidance, which aims to reduce some health problems caused by poor sitting postures by guiding the person to the correct posture. Morrell and Wasilewski [15] proposed a system that provides vibrotactile feedback as an ambient sensation that is consistently present. Their objective was not to show faster reaction times to a single hazard but to show better management of risk. The system transmits feedback continuously to the driver's body on a location that is physically close to the object to provide better awareness of the environment.

Although these studies involved sitting postures and vibrotactile warning systems, a complete system that fully integrates both in a realistic setting does not exist. Moreover, most of the presented studies did not provide a detailed evaluation or report the accuracy criteria of posture detection. In this study, a complete system for the design and implementation of sitting postures recognition that is integrated into the fabric of a real driving seat is proposed. No previous study reported such realistic integration. Furthermore, the proposed method introduces the placement of the force sensors inside the fabric of the seat. Moreover, a state-of-art virtual reality driving simulator that provides a realistic environment to test the system with different scenarios was used.

\section{System Architecture}

In the proposed system, if a potential conflict is detected, a vibration is emitted depending on the risk assessment of the condition. The system provides three levels of action. The first action is to provide no alert in case of no conflict or case of the driver making a proper response (braking, decelerating, etc.). If the response of the driver is not enough or the driver does not take any corrective action, the intensity of the vibration will increase. Fig. 1 shows the high-level architecture of the proposed system.

To replicate the proposed system in the lab, a driving set from LogitechTM was used to provide a natural interface for the driver, including the steering wheel, gearshift, and pedals to generate triggers as an indication of the car's direction, speed, and acceleration. Two sensor mats were designed to detect the sitting postures. The seat also included vibrators as well to provide warning signals. The hardware is controlled by Arduino Mega 2560 microcontroller. The controller receives the signal from the software side wirelessly via Bluetooth connection to read the data of the force sensors and trigger the vibration according to the events triggered in the scenario inside the driving simulation while driving. 


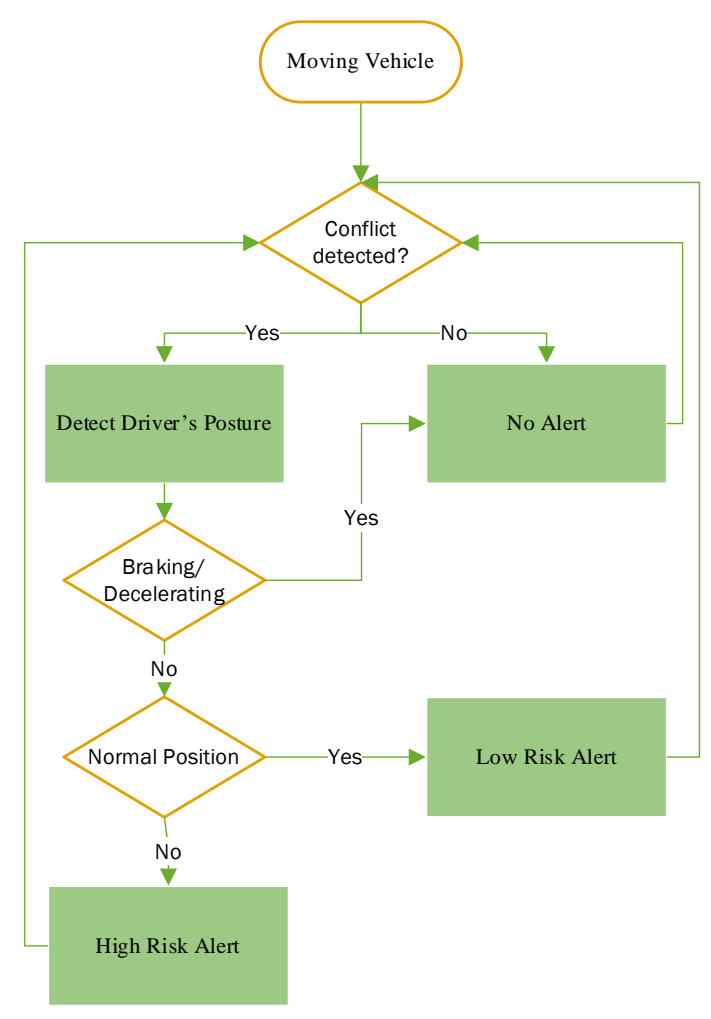

Fig. 1: High-level Architecture of the Proposed System.

\subsection{Driver Activity Recognition (DAR)}

The proposed system components are shown in Fig. 2. The DAR is the main functional unit in this system. It performs the process of analyzing the data received from the sensors to recognize the posture of the driver. This unit is also responsible for deciding whether the driver needs to be alerted or not based on the detected posture. To recognize the type of posture, the different types of postures are stored and tagged in the system as "expected" from the simulator environment part. When the simulator sends a signal to indicate this type of expected posture, it will be compared with the signal coming from the DAR section. Depending on the comparison, a signal that represents the decision is sent to the hardware engine.

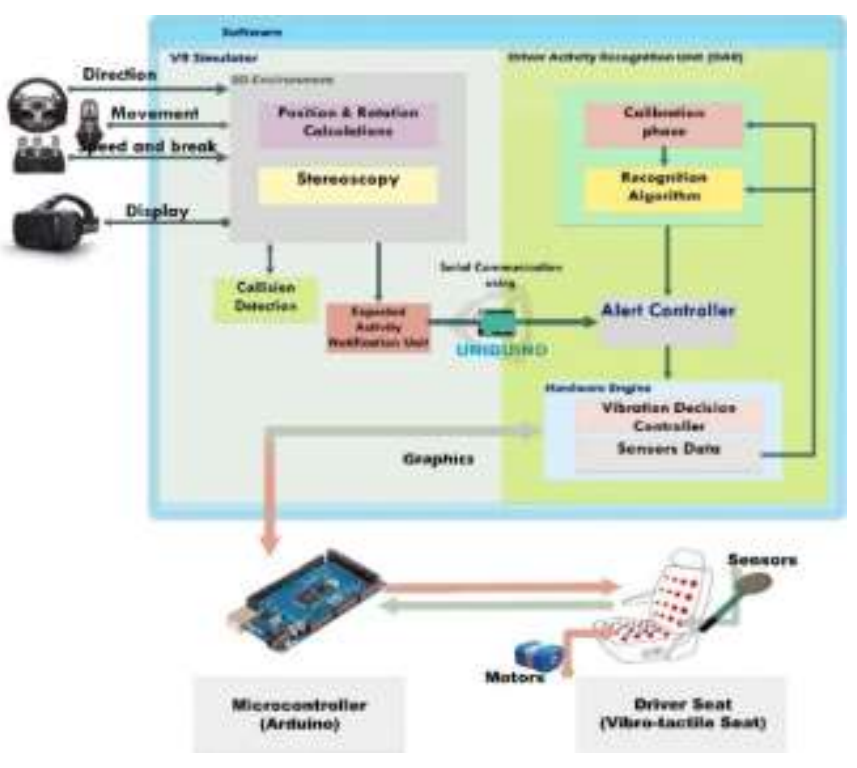

Fig. 2. Proposed System Components [16].

\subsection{Seat Design}

The seat incorporates force sensors for posture recognition. Force sensors are placed on both the backrest and the pan seat to acquire the driver's posture. One novel aspect in the hardware design is the neat placement of the force sensors in the fabric of the seat which resembles a realistic setting. Furthermore, the number of sensors is relatively large, which can cover all the seat area and can contribute more to the enhancement of accuracy. The distribution of the force sensors on the seat and backrest of the driver was carefully selected. A total of 16 force sensors $(4 \times 4)$ were placed on the backrest, and 20 force sensors were placed on the seat. They were placed in certain locations after testing different methods as discussed later. To be able to connect the 36 sensors to the Arduino microcontroller limited available inputs, 32 sensors were connected to two Analog/Digital multiplexers (16 sensors per 1 multiplexer) and the rest of the sensors were connected directly to the Arduino microcontroller. Multiplexers were used due to the limited input analog pins in Arduino Mega 2560 microcontroller.

\subsection{Distribution of Sensors}

The allocation of the sensors was selected based on several tests carried out to choose the most suitable distribution. The initial idea was to distribute the sensors in an array of $16 \times 16$ in both the seat and the backrest as can be seen in Fig. 3 (Left). The second approach was to divide the distribution of sensors into 8 areas as shown in Fig. 3 (Middle). The threshold of readings in each area was computed using several ways, including mean squared error, entropy, and normal average. All the previous algorithms were tested thoroughly; the result was surprisingly less accurate than the basic distribution without any division or specific computation. Based on this, the first simple distribution approach was adopted. During the testing, it was noticed that the basic distribution resulted in good accuracy for all the postures except for the acceleration and brake postures. This is because the body is not moving and only the right leg and in fine movements during these actions. To solve this issue, four more sensors were added to the area of the seat where the pressure is 
concentrated while performing acceleration and brake postures as shown in Fig. 3 (Right).
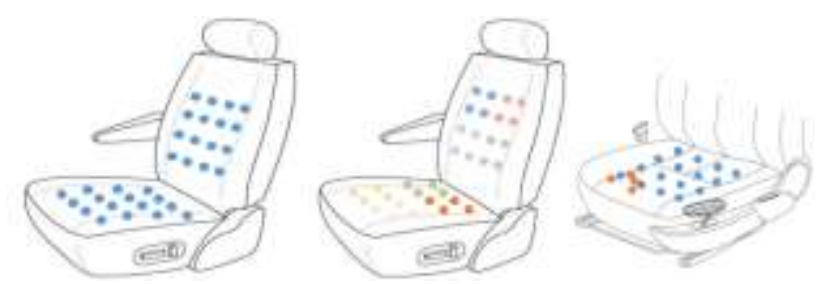

Fig. 3. Distribution of Force Sensors Using Normal Distribution Without Grouping or Dividing into Areas (Left), Distribution of Force Sensors by Dividing Sensors into Areas According to Different Approaches (Middle), and Distribution of Sensors with the Addition of Four Additional Sensors for Brake and Accelerate Posture (Right) [16]

\section{Posture Recognition Algorithm}

The software structure of the simulation is divided into a 3D driving environment and recognition algorithm. The 3D environment was developed using Unity3D. It simulates the driving process and includes scenarios to test the system which in our case was to test the scenario of a pedestrian crossing the road. The algorithm has two phases:

(1) Calibration: Drivers are diverse and may vary in weight, height, and sitting posture. The proposed solution is to have the seat calibrated for the first time to the user. The calibration process was simply asking the user to sit in the driving seat and make each posture. The data reading for each posture was recorded and used as a baseline in the recognition stage for this specific driver.

(2) Recognition: According to calibration readings, the recognition is decided based on a simple comparison between the current reading of the sensors and the saved calibrated data. The final posture is then decided based on the least difference between the reading and the different postures in the calibrated data. Fig. 4 shows the steps of a posture detection algorithm.

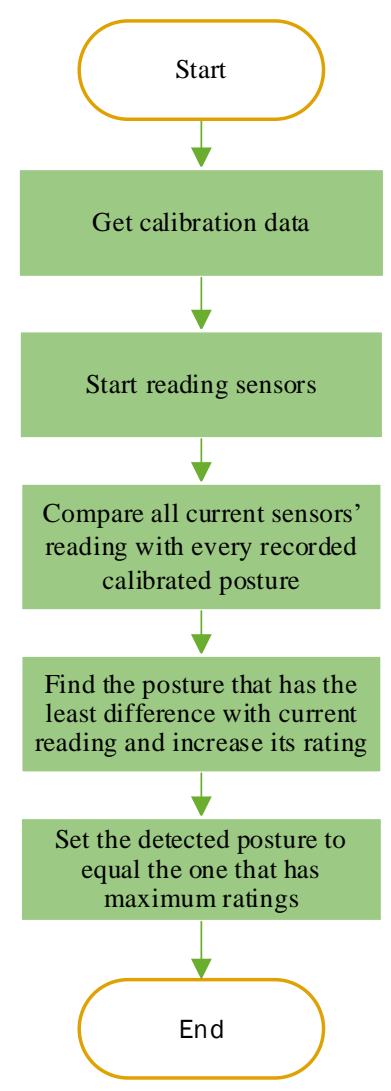

Fig. 4. Flowchart of the Detection Algorithm Steps.

\section{Experiment and Results}

\subsection{Simulation Development}

To evaluate the effectiveness of the designed seat and algorithm in posture recognition, a virtual reality driving simulation environment was developed as an attempt to recreate a realistic driving workload and provide a somehow realistic environment. State-of-art VR Oculus Rift head-mounted display (HMD) was used to create an immersive experience which is an essential element for achieving a realistic result as the sense of presence can affect the reaction. Unity3D game engine was used to develop the 3D graphics driving simulation and for the integration of HMD. Furthermore, it was used in the integration of the Arduino microcontroller to be able to communicate with the force sensors and vibrators. The car used for the simulation had a full detailed interior to be able to simulate different situations in which the driver conducts different activities inside the car. This makes the whole simulation realistic as the driver can look around and feels like he/she is inside the car.

\subsection{Sample Characteristics}

The proposed system was initially tested using a sample of 24 female drivers, and initial results were obtained [16]. For the final analysis, an additional 30 male drivers participated in the study. Table 1 shows the distribution of the participants according to different height and weight categories.

Table 1. Number of Participants Based on Different Height and Weight Categories.

\begin{tabular}{llllllll}
\hline Weight & Height & Less & $165-$ & $170-$ & 175 & or & Tota \\
\hline & Count & 7 & 6 & 0 & 1 & 14 \\
\hline
\end{tabular}




\begin{tabular}{|c|c|c|c|c|c|c|}
\hline Less & $\%$ & $13.0 \%$ & 11.1 & $0.0 \%$ & $1.9 \%$ & 25.9 \\
\hline \multirow{2}{*}{$\begin{array}{l}60- \\
69.9\end{array}$} & Count & 2 & 6 & 3 & 2 & 13 \\
\hline & $\%$ of & $3.7 \%$ & 11.1 & $5.6 \%$ & $3.7 \%$ & 24.1 \\
\hline \multirow{2}{*}{$\begin{array}{l}70- \\
79.9\end{array}$} & Count & 0 & 2 & 5 & 4 & 11 \\
\hline & $\%$ of & $0.0 \%$ & $3.7 \%$ & $9.3 \%$ & $7.4 \%$ & 20.4 \\
\hline \multirow{2}{*}{$\begin{array}{l}80 \text { or } \\
\text { more }\end{array}$} & Count & 1 & 1 & 5 & 9 & 16 \\
\hline & $\%$ & $1.9 \%$ & $1.9 \%$ & $9.3 \%$ & $16.7 \%$ & 29.6 \\
\hline \multirow[t]{2}{*}{ Total } & Count & 10 & 15 & 13 & 16 & 54 \\
\hline & $\%$ & $18.5 \%$ & 27.8 & 24.1 & $29.6 \%$ & 100. \\
\hline
\end{tabular}

To assess how efficient the system is in satisfying the functional requirements, several tests were conducted using the virtual reality simulation. The experiments were designed to test the recognition accuracy for nine postures; normal sitting, accelerating, braking, leaning right, leaning right forward, leaning right backward, leaning left, leaning left forward, and stretching-up. The experimental setup started with the calibration process. In this process, each driver was asked to perform the different postures that the system can detect. The data were then saved for this specific user. The second phase is driving according to a certain scenario. During this time, each participant was asked to perform different tasks (pick up objects, look backward, drive naturally, etc.). Each posture was then compared with the corresponding recognized one from the system.

\subsection{Recognition Accuracy}

The experiment was conducted in two modes, (1) with virtual reality (with-VR) and (2) without it (without-VR). In withoutVR mode, a real physical environment was provided using real driving scenarios. The subjects went first through a calibration test, then they were asked to perform some tasks to illustrate each posture. The data were recorded, and the accuracy of each detected posture was calculated. The results of accuracy for each posture can be seen in Table 2. The developed system was able to recognize all nine postures with an average accuracy of $92 \%$ in the case of testing without VR. In the case of testing with-VR mode, the users wear the HMD and were asked to drive in the virtual reality driving simulator.

The subjects were given sufficient time to familiarize themselves with the environment until they feel comfortable to reduce the possible negative effects of learnability on the result. The postures were observed, and accuracy was calculated for each posture.

Table 2 shows the accuracy for each posture in with-VR mode. In this case, the average accuracy increased slightly to $92.4 \%$. This might be because the VR simulation provided a realistic environment and motivated the subject to react naturally that may lead to an accurate reaction. Furthermore, it might be because the collected calibration data was improved, which also lead to an improvement in the accuracy. 
Table 2. Recognition Accuracy for the Cases of Without VR testing mode and With-VR testing mode. testing. With the advancement of display technology, a realistic simulation with high quality and low cost at the same time was achieved. The recognition algorithm accuracy varied from $85.2 \%$ to $100 \%$. Postures' accuracy might improve by improving

\begin{tabular}{|c|c|c|c|c|c|c|c|c|c|c|c|c|c|c|c|c|c|c|c|c|}
\hline \multicolumn{2}{|c|}{ Testing Without-VR } & \multirow[t]{2}{*}{$\begin{array}{l}\text { To } \\
\text { tal }\end{array}$} & \multicolumn{2}{|c|}{$\begin{array}{l}\text { Normal } \\
\text { Sitting }\end{array}$} & \multicolumn{2}{|c|}{ Accelerate } & \multicolumn{2}{|c|}{ Break } & \multicolumn{2}{|c|}{ Lean Right } & \multicolumn{2}{|c|}{$\begin{array}{l}\text { Lean Right } \\
\text { Forward }\end{array}$} & \multicolumn{2}{|c|}{$\begin{array}{c}\text { Lean Right } \\
\text { Backward }\end{array}$} & \multicolumn{2}{|c|}{ Lean Left } & \multicolumn{2}{|c|}{$\begin{array}{c}\text { Lean Left } \\
\text { Forward }\end{array}$} & \multicolumn{2}{|c|}{ Stretch-up } \\
\hline & & & $\mathrm{N}$ & $\%$ & $\mathrm{~N}$ & $\%$ & $\mathrm{~N}$ & $\%$ & $\mathrm{~N}$ & $\%$ & $\mathrm{~N}$ & $\%$ & $\mathrm{~N}$ & $\%$ & $\mathrm{~N}$ & $\%$ & $\mathrm{~N}$ & $\%$ & $\mathrm{~N}$ & $\%$ \\
\hline \multirow[t]{3}{*}{ Gender } & Male & 34 & 34 & 100 & 29 & 85.3 & 30 & 88.2 & 29 & 85.3 & 31 & 91.2 & 30 & 88.2 & 32 & 94.1 & 32 & 94.1 & 31 & 91.2 \\
\hline & Female & 20 & 20 & 100 & 18 & 90.0 & 18 & 90.0 & 17 & 85.0 & 19 & 95.0 & 19 & 95.0 & 19 & 95.0 & 19 & 95.0 & 20 & 100 \\
\hline & Less than 60 & 14 & 14 & 100 & 12 & 85.7 & 14 & 100 & 10 & 71.4 & 14 & 100 & 14 & 100.0 & 13 & 92.9 & 13 & 92.9 & 14 & 100 \\
\hline \multirow{4}{*}{$\begin{array}{l}\text { Weight } \\
(\mathrm{kg})\end{array}$} & $60-69.9$ & 13 & 13 & 100 & 11 & 84.6 & 11 & 84.6 & 12 & 92.3 & 12 & 92.3 & 13 & 100 & 13 & 100 & 13 & 100 & 12 & 92.3 \\
\hline & $70-79.9$ & 11 & 11 & 100 & 10 & 90.9 & 10 & 90.9 & 10 & 90.9 & 9 & 81.8 & 9 & 81.8 & 10 & 90.9 & 9 & 81.8 & 10 & 90.9 \\
\hline & 80 or more & 16 & 16 & 100 & 14 & 87.5 & 13 & 81.3 & 14 & 87.5 & 15 & 93.8 & 13 & 81.3 & 15 & 93.8 & 16 & 100 & 15 & 93.8 \\
\hline & Less than 165 & 10 & 10 & 100 & 8 & 80.0 & 10 & 100.0 & 9 & 90.0 & 10 & 100 & 10 & 100 & 10 & 100 & 10 & 100 & 10 & 100 \\
\hline \multirow{3}{*}{$\begin{array}{l}\text { Height } \\
(\mathrm{cm})\end{array}$} & $165-169.9$ & 15 & 15 & 100 & 12 & 80.0 & 12 & 80.0 & 12 & 80.0 & 13 & 86.7 & 14 & 93.3 & 13 & 86.7 & 13 & 86.7 & 14 & 93.3 \\
\hline & $170-174.9$ & 13 & 13 & 100 & 12 & 92.3 & 12 & 92.3 & 10 & 76.9 & 13 & 100 & 12 & 92.3 & 13 & 100 & 12 & 92.3 & 12 & 92.3 \\
\hline & 175 or more & 16 & 16 & 100 & 15 & 93.8 & 14 & 87.5 & 15 & 93.8 & 14 & 87.5 & 13 & 81.3 & 15 & 93.8 & 16 & 100 & 15 & 93.8 \\
\hline Average & 92.0 & 54 & 54 & 100 & 47 & 87.0 & 48 & 88.9 & 46 & 85.2 & 50 & 92.6 & 49 & 90.7 & 51 & 94.4 & 51 & 94.4 & 51 & 94.4 \\
\hline
\end{tabular}

\begin{tabular}{|c|c|c|c|c|c|c|c|c|c|c|c|c|c|c|c|c|c|c|c|c|}
\hline \multicolumn{21}{|c|}{ Testing With-VR } \\
\hline Gender & Male & 34 & 34 & 100 & 29 & 85.3 & 28 & 82.4 & 29 & 85.3 & 33 & 97.1 & 30 & 88.2 & 34 & 100 & 34 & 100 & 29 & 85.3 \\
\hline & Female & 20 & 20 & 100 & 18 & 90.0 & 19 & 95.0 & 18 & 90.0 & 18 & 90.0 & 18 & 90.0 & 19 & 95.0 & 19 & 95.0 & 20 & 100 \\
\hline \multirow{4}{*}{$\begin{array}{l}\text { Weight } \\
(\mathrm{kg})\end{array}$} & Less than 60 & 14 & 14 & 100 & 13 & 92.9 & 14 & 100 & 12 & 85.7 & 12 & 85.7 & 13 & 92.9 & 13 & 92.9 & 13 & 92.9 & 13 & 92.9 \\
\hline & $60-69.9$ & 13 & 13 & 100 & 11 & 84.6 & 11 & 84.6 & 12 & 92.3 & 13 & 100 & 12 & 92.3 & 13 & 100 & 13 & 100 & 12 & 92.3 \\
\hline & $70-79.9$ & 11 & 11 & 100 & 10 & 90.9 & 9 & 81.8 & 8 & 72.7 & 11 & 100 & 10 & 90.9 & 11 & 100 & 11 & 100 & 9 & 81.8 \\
\hline & 80 or more & 16 & 16 & 100 & 13 & 81.3 & 13 & 81.3 & 15 & 93.8 & 15 & 93.8 & 13 & 81.3 & 16 & 100 & 16 & 100 & 15 & 93.8 \\
\hline \multirow{4}{*}{$\begin{array}{l}\text { Height } \\
(\mathrm{cm})\end{array}$} & Less than 165 & 10 & 10 & 100 & 10 & 100 & 10 & 100 & 8 & 80.0 & 9 & 90.0 & 9 & 90.0 & 10 & 100 & 10 & 100 & 10 & 100 \\
\hline & $165-169.9$ & 15 & 15 & 100 & 12 & 80.0 & 12 & 80.0 & 12 & 80.0 & 14 & 93.3 & 14 & 93.3 & 14 & 93.3 & 14 & 93.3 & 14 & 93.3 \\
\hline & $170-174.9$ & 13 & 13 & 100 & 12 & 92.3 & 11 & 84.6 & 12 & 92.3 & 13 & 100 & 11 & 84.6 & 13 & 100 & 13 & 100 & 12 & 92.3 \\
\hline & 175 or more & 16 & 16 & 100 & 13 & 81.3 & 14 & 87.5 & 15 & 93.8 & 15 & 93.8 & 14 & 87.5 & 16 & 100 & 16 & 100 & 13 & 81.3 \\
\hline $\begin{array}{l}\text { Average } \\
(\%)\end{array}$ & 92.4 & 54 & 54 & 100 & 47 & 87.0 & 47 & 87.0 & 47 & 87.0 & 51 & 94.4 & 48 & 88.9 & 53 & 98.1 & 53 & 98.1 & 49 & 90.7 \\
\hline
\end{tabular}

$(\%)$

\section{N: Number of Recognized Postures \\ $\%$ : Percentage of Recognized Postures}

\section{Conclusion}

In this study, a system for sitting postures recognition was proposed and tested. The results showed that drivers' postures can be considered as a vital and informative source that can help in identifying the driver's activities. The developed system was able to accurately recognize different postures including normal sitting, accelerating, braking, leaning right, leaning right forward, leaning right backward, leaning left, leaning left forward, and stretching up. The study also presented an immersive virtual reality driving simulation that can be used for the calibration process and the proposed algorithm itself. Therefore, more investigation has to be carried out on how to improve the calibration and reduce its effect on the recognition rate. Nevertheless, the current recognition ratio is considered high. In addition, additional work on improving the detection rate for some postures using different algorithms should be considered. Furthermore, developing a more user-friendly interface for the calibration phase where $3 \mathrm{D}$ visuals can be provided for each posture is needed as this may lead to better accuracy as the user will be able to do the calibration more easily and accurately. Finally, the proposed system can be used to alert drivers to disengage autonomous mode in the case of selfdriving and autonomous vehicles when conditions are unsafe. 


\section{Acknowledgment}

This paper was made possible by a UREP award [UREP18-1612-064] from the Qatar National Research Fund (a member of The Qatar Foundation). The statements made herein are solely the responsibility of the authors.

\section{References}

1. Wen, H., et al., Effect of music listening on physiological condition, mental workload, and driving performance with consideration of driver temperament. International journal of environmental research and public health, 2019. 16(15): p. 2766.

2. Prat, F., et al., Driving distractions: An insight gained from roadside interviews on their prevalence and factors associated with driver distraction. Transportation research part F: traffic psychology and behaviour, 2017. 45: p. 194-207.

3. Shaaban, K. and K. Abdelwarith, Understanding the association between cell phone use while driving and seat belt noncompliance in Qatar using logit models. Journal of Transportation Safety \& Security, 2020. 12(2): p. 292308.

4. Shaaban, K., S. Gaweesh, and M. Ahmed, Characteristics and Mitigation Strategies for Cell Phone Use While Driving Among Young Drivers in Qatar. Journal of Transport \& Health, 2018.

5. Shaaban, K., S. Gaweesh, and M.M. Ahmed, Investigating in-vehicle distracting activities and crash risks for young drivers using structural equation modeling. PLoS one, 2020. 15(7): p. e0235325.

6. Mota, S. and R.W. Picard. Automated posture analysis for detecting learner's interest level. in 2003 Conference on Computer Vision and Pattern Recognition Workshop. 2003. IEEE.

7. Zhu, M., A.M. Martinez, and H.Z. Tan. Template-based recognition of static sitting postures. in 2003 Conference on Computer Vision and Pattern Recognition Workshop. 2003. IEEE.

8. Riener, A. and A. Ferscha, Driver activity recognition from sitting postures. Mensch \& Computer 2007 Workshopband, 2007.

9. Johnson, D.A. and M.M. Trivedi. Driving style recognition using a smartphone as a sensor platform. in 2011 14th International IEEE Conference on Intelligent Transportation Systems (ITSC). 2011. IEEE.

10. Riener, A., A. Ferscha, and M. Matscheko. Intelligent vehicle handling: Steering and body postures while cornering. in International Conference on Architecture of Computing Systems. 2008. Springer.
11. Higuchi, M. and P. Raksincharoensak. Seat vibrotactile warning interface for forward vehicle collision avoidance. in Proceedings of SICE Annual Conference 2010. 2010. IEEE.

12. Van Erp, J.B. and H.A. Van Veen, Vibrotactile in-vehicle navigation system. Transportation Research Part F: Traffic Psychology and Behaviour, 2004. 7(4-5): p. 247-256.

13. Riener, A. and A. Ferscha. Reconfiguration of vibrotactile feedback based on drivers' sitting attitude. in 2009 Second International Conferences on Advances in Computer-Human Interactions. 2009. IEEE.

14. Zheng, Y. and J.B. Morrell. A vibrotactile feedback approach to posture guidance. in 2010 IEEE haptics symposium. 2010. IEEE.

15. Morrell, J. and K. Wasilewski. Design and evaluation of a vibrotactile seat to improve spatial awareness while driving. in 2010 IEEE Haptics Symposium. 2010. IEEE.

16. Halabi, O., et al. Driver activity recognition in virtual reality driving simulation. in 2017 8th International Conference on Information and Communication Systems (ICICS). 2017. IEEE. 